\title{
A Survey and Evaluation of the Potentials of Distributed Ledger Technology for Peer-to-Peer Transactive Energy Exchanges in Local Energy Markets
}

\author{
Pierluigi Siano ${ }^{\circledR}$, Senior Member, IEEE, Giuseppe De Marco, Member, IEEE, \\ Alejandro Rolán ${ }^{(0)}$, and Vincenzo Loia ${ }^{\circledR}$, Senior Member, IEEE
}

(Survey Tutorial)

\begin{abstract}
The unpredictability and intermittency introduced by Renewable Energy Sources (RESs) in power systems may lead to unforeseen peaks of energy production, which might differ from energy demand. To manage these mismatches, a proper communication between prosumers (i.e., users with RESs that can either inject or absorb energy) and active users (i.e., users that agree to have their loads changed according to the system needs) is required. To achieve this goal, the centralized approach used in traditional power systems is no longer possible because both prosumers and active users would like to take part in energy transactions, and a decentralized approach based on transactive energy systems (TESs) and Peer-to-Peer (P2P) energy transactions should be adopted. In this context, the Distributed Ledger Technology (DLT), based on the blockchain concept arises as the most promising solution to enable smart contracts between prosumers and active users, which are safely guarded in blocks with cryptographic hashes. The aim of this paper is to provide a review about the deployment of decentralized TESs and to propose and discuss a transactive management infrastructure. In this context, the concept of Proof of Energy is proposed as a novel consensus protocol for P2P energy exchanges managed by DLT. An application of the proposed infrastructure considering a Virtual Power Plant (VPP) aggregator and residential prosumers endowed with a new transactive controller to manage the electrical storage system is discussed.
\end{abstract}

Index Terms-Aggregator, battery, blockchain, demand response, distributed ledger technology, local energy market, microgrid, peer-to-peer transactions, prosumer, smart contracts, smart grid, transactive energy.

\section{NOMENCLATURE}

AnC Analytics component.

BFT Byzantine fault tolerant.

CHP Combined heat and power.

CPF Consumption-production function.

Manuscript received November 20, 2018; revised February 1, 2019; accepted March 2, 2019. This work was supported in part by the Spanish Ministry of Education, Culture, and Sport under Grant CAS17/00438. (Corresponding author: Pierluigi Siano.)

P. Siano, G. De Marco, and V. Loia are with the Department of Management and Innovation Systems, University of Salerno, Fisciano 84084, Italy (e-mail: psiano@unisa.it; demarco.giu@gmail.com; loia@unisa.it).

A. Rolán is with the Department of Industrial Engineering, Ramon Llull University, Barcelona 08017, Spain (e-mail: alejandro.rolan@iqs.url.edu).

Digital Object Identifier 10.1109/JSYST.2019.2903172
CSM Certified smart meter. 40

DER Distributed energy resource.

DLC Direct load control.

DLT Distributed ledger technology.

DR Demand response.

DSM Demand-side management.

DSO Distribution system operator.

EMS Energy management system.

EV Electric vehicle

GWAC GridWise architecture council.

HDG Home device gateway.

HEM Home energy manager.

HVAC Heating, ventilation, and air conditioning.

IAS Immutability, anonymity, and security.

IB Information backbone.

ICT Information and communication technology.

IoT Internet of Things.

JSON JavaScript object notation.

LEM Local energy market.

MQTT Message queue telemetry transport.

MV Mid voltage.

P2P Peer to peer.

PNWSGD Pacific northwest smart grid demonstration.

PoE Proof of energy.

PoS Proof of stake.

PoW Proof of work.

PV Photovoltaic.

QoS Quality of service.

REonly Rewards only.

RES Renewable energy source.

SOC State of charge.

TC Transactive control.

TCR Transactive controller.

TE Transactive energy.

TEonly Transactive energy only.

TES Transactive energy system.

TMI Transactive management infrastructure.

TMP Transactive management platform. 
78 VPP

79 WT
Virtual power plant.

Wind turbine.

\section{INTRODUCTION}

D URING the last decades, distributed generation units based on Renewable Energy Sources (RES) have been integrated into electrical grids, mainly at the distribution level. By the end of 2016, around 25\% of the electricity production around the world originated from RES [1], with wind energy, bio-power energy, and solar photovoltaic (PV) being the energy pivotal sources. Studies reveal that in the next years there will be a major increase in the penetration of RES into the grid, which will reach a share around $30 \%$ by 2022 [2] and this share will even exceed $60 \%$ by 2050 [3]. Moreover, since the installation of Distributed Energy Resources (DERs), including PV panels, micro Wind Turbines (WTs), diesel, and bio-generators has become an affordable investment in many countries, from the point of view of both residential and business users, they have been deployed at the consumption side too [4]. At the same time, electric loads are not "dump" anymore. They can auto-regulate the power absorption, intelligently and/or by responding to external Demand Response (DR) signals from the grid. In recent years, the concepts of Demand-Side Management (DSM) and DR have arisen in order to balance energy generation with energy consumption [5] and help preventing the congestion problems [6]. By applying a DR approach, end users under incentive-based programs let suppliers to control all or some of their loads by means of Direct Load Control (DLC) [7]. For example, during peak hours, users may receive incentives to have their loads reduced, such as Heating, Ventilation, and Air Conditioning (HVAC) loads [8]. To do so, smart metering based on Internet of Things (IoT) is required in order to send real-time data among all users [9], [10]. Therefore, the overall network becomes a smart grid, as there is not only a flow of energy but also a flow of data, which managed properly, will determine an efficient distribution of energy through the entire system [11], [12]. Both DERs and DR have opened new opportunities for the power grid, and new challenges as well, because DERs are intermittent and nonuniformly deployed. Opportunities have arisen regarding the optimization of power flows, the improvement of the stability of the power grid, and the reduction of the economic impact of production and deployment of energy reserves. On the other hand, the energy market regulation and the management of energy trading also represent a concern.

In recent years, in order to exploit all the benefits of DERs and to meet policies and targets toward decarbonisation, a new kind of paradigm has been proposed. This paradigm is based on two key concepts, namely: Transactive Energy (TE) and peer-to-peer (P2P) management by means of Distributed Ledger Technology (DLT), based on blockchain. These concepts will be carefully addressed in the following sections.

As it will be better described and detailed in the next sections, the contributions of this paper are mainly related to, shown as follows.

1) The survey and assessment of the potentials of DLT for P2P Transactive Energy Exchanges in Local Energy Markets.
2) The detailed description of a new transactive management 134 infrastructure, based on DLT, implementing a TE system 135 leveraging P2P energy exchanges (defined here as P2P- 136 TE) in Local Energy Markets (LEMs).

3) The proposal of an innovative Proof of Energy (PoE) func- 138 tion as a candidate of the consensus protocol for P2P 139 energy exchanges managed by DLT. The proposed con- 140 sensus protocol is not energy demanding as in other per- 141 missioned DLT and it is able to promote a social behavior 142 based on sustainable and circular economy. 143

4) The proposal of an innovative transactive controller (TCR) 144 to manage the operation of the battery of a residential 145 prosumer.

The paper is structured as follows. Sections II and III give a 147 survey on Local Energy Markets (LEM) and Transactive Energy 148 Systems (TESs), respectively. Section IV reviews and discuss 149 the concept of blockchain-based DLT applied to TESs. Finally, 150 in Section VI, a novel transactive management infrastructure 151 (TMI) to enable P2P energy exchanges among all the grid- 152 connected users is proposed and described. An innovative Proof 153 of Energy (PoE) function is also proposed in order to implement 154 P2P energy exchanges based on DLT in a LEM context.

\section{LOCAL ENERGy MARKETS AND THE ROLE OF PROSUMERS}

The electricity market is defined by both entire market and 157 sub market [13]. The former is based on end-product markets 158 and intermediate-product markets, while the latter includes the 159 wholesale market and those for ancillary services. A Local En- 160 ergy Market (LEM) can be seen as a kind of sub-market, where 161 participants can be aggregated for flexibility purposes [14] such 162 as constraints management, portfolio optimization and system 163 balancing in order to balance demand and supply. The current 164 research activities on LEMs are related on market mechanisms 165 [15], agent preferences and strategies [16] and transactional 166 product of reserve energy [17]. The presented paper fits in the 167 latter two topics.

In order to integrate a LEM into the entire market, differ- 169 ent organizational models for flexibility management have been 170 compared in [18] for both Germany and the Netherlands. This 171 paper reveals that the dynamic pricing and local aggregator ap- 172 proaches work properly in the retail market. In [19] the use of 173 a LEM is proposed to secure the integration of large renewable 174 energy systems into the main energy system. The study devel- 175 oped in [20] proves that it is feasible to include Combined Heat 176 and Power (CHP) plants to help balancing the fluctuation of 177 wind power systems. Both [19], [20] focus their study on some 178 examples in Denmark.

A current example of a LEM is furnished in the empower 180 project [21], which does not focuses on price but on a value- 181 oriented approach. It can be used to carry out different contracts 182 among partners, such as cross-subsidized energy contracts or 183 flexibility contracts. Some pilot tests combining the Empower 184 concept and the real-time shared knowledge about energy needs 185 among households and communities have been proved in Nor- 186 way, Germany, and Malta with promising results. Another ex- 187 ample is the design of a LEM which has been developed by 188 the Chalmers University of Technology in Sweden for the cam- 189 


\section{A. Transactive Energy Concept} LEM [23].

\section{B. Transactive Controllers} energy requirements. pus itself [22]. The computational model in this project was validated by experimental results and it was concluded that the LEM was not able to provide itself the required energy, thus requiring external energy resources.

Let us consider, for example, a community or a set of communities of users who can arbitrarily belong to one of the following categories: 1) prosumers, i.e., users who provide the grid with locally generated electrical energy, such as PV, WT or diesel generators, that can either inject or absorb electrical energy; 2) active users with flexible and shiftable loads, electric vehicles (EVs) and HVAC systems that may be controlled by DR signals managed by TCRs; and 3) passive users, not participating in any DR program. These communities are usually organized geographically and, from the grid's viewpoint, they are attached to a common node in a distribution bus and can participate in a

In a LEM, whenever the net consumed energy is positive, prosumers can decide to sell part of the produced energy. In this way, a surplus of energy in the grid may exist. On the other hand, active users can "buy" this surplus by regulating their loads. Active users can also virtually sell energy, by responding to DR signals and reducing or time-shifting their electrical loads [24]. It should be noted that this trading must be done in a secure and privacy-preserving way, as the transactions in a LEM are carried out in a decentralized way. This can be achieved by a proper bidding algorithm with privacy-preserving protocols [25].

As previously stated, in the new grid scenario the consumers with the ability to inject energy into the grid (prosumers) would also like to take part in the electricity market by maximizing their profits while delivering energy and minimizing their costs when absorbing it [26]. In other words, a two-way grid management is required in order to enable energy transactions among all the participants [27]. In this context, the GridWise Architecture Council (GWAC) [28] has proposed the following definition for Transactive Energy (TE): "a system of economic and control mechanisms that allows the dynamic balance of supply and demand across the entire electrical infrastructure using value as a key operational parameter." The concept of "value" is related to the definition of price and incentives in order to guarantee that all DER owned by prosumers generate and consume electricity in a win-win approach [29], i.e., by looking for the benefits of all the users and not for the benefit of one or a few ones.

A classification of control strategies in smart grids identifies passive, active, interactive, and TCRs [30]. While passive controllers operate without exchanging information with the utility, active controllers enable customers to adjust their energy consumption depending on price changes. TCRs represent the most promising evolution for energy users aiming at participating in LEMs since they allow both prosumers and active users to make bids considering the real time price of electrical energy and their

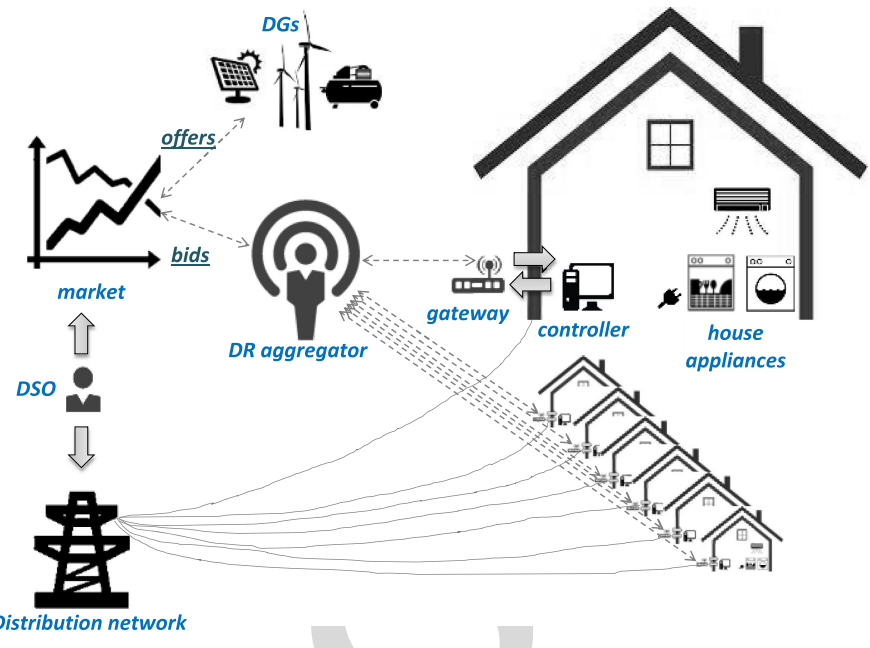

Fig. 1. System architecture adopted for TCRs' operation [33].

A TCR is basically implemented on an energy gateway which 244 communicates over the Information Backbone (IB), e.g., the 245 Internet, to exchange all the information needed to trade and 246 manage energy transactions [31]. In other words, the TCR acts 247 as a negotiator of energy prices on the energy market in order 248 to modify the user's settings according to price signals, which 249 perfectly fits with the concept of LEM, explained in Section III. 250 The TCR is used also to grant access to the information platform 251 used to coordinate the signing of smart contracts and the record- 252 ing of energy transaction. Finally, the TCR communicates also 253 to smart devices used by active users, which can be sensors or 254 actuators, such as smart meters, smart lamps and HVACs. 255

A transactive scheme is proposed in [32], where a distributed 256 iterative algorithm for optimal demand in residential applica- 257 tions is developed. Another example is given in [33], where a 258 TCR and some smart plugs-in for some electrical loads are 259 adopted. In this approach, a residential energy gateway ex- 260 changes information with a DR aggregator, which is in charge of 261 different houses. Each DR aggregator sets the bids for electrical 262 energy according to the signals received from the TCRs of each 263 house and gives feedback signals (acceptance or rejection of a 264 bid) to the corresponding TCRs. The described architecture is 265 depicted in Fig. 1.

Among the three levels of control in electrical grids, i.e., pri- 267 mary control for dynamic response (milliseconds), secondary 268 control for frequency control (seconds) and tertiary control 269 (minutes) over the whole system, this last control acts in order to 270 balance energy generation and energy consumption combined 271 with economic signals, thus also contributing to frequency con- 272 trol in a social-welfare maximization approach [34]. In order to 273 implement a proper Transactive Control (TC), distributed intel- 274 ligent devices based on Information and Communication Tech- 275 nology (ICT), such as smart meters, must be used [35], [36]. 276 Smart meters enable exchanging energy data in near-real-time 277 fashion among all the agents (prosumers and active users). $\quad 278$

When a prosumer wishes to make either a demand (buy) or 279 an offer (sell) of energy from/to the grid (which is recorded by a 280 smart metering), it sends its request to a parent node, where other 281 prosumers are connected. Then, a TC-based platform evaluates 282 
all bids from all the energy-demand prosumers, assesses the offers from energy-production prosumers and finally sends the corresponding prices to all the prosumers, which can either accept or reject the transactions [37].

For the last few years, some pilot projects have been carried out on the topic of managing small communities under a TES perspective, showing promising results. For example, in the USA, the Pacific Northwest Smart Grid Demonstration Project (PNWSGD) [38] has coped with the grid congestion problem by acting on smart thermostats for HVACs control, while in the Netherlands the PowerMatching City Project [39] has dealt with supply and demand management issues in the first smart grid village in Europe. More examples of TE-based pilot projects and their comparison can be found in [31] and [36].

\section{Distributed Ledger TeChNOlogy FOR TRANSACtiVE ENERGY SYSTEMS}

\section{A. Advantages of a Peer-to-Peer Decentralized} Architecture for Energy Exchanges

In traditional power systems, every transaction is centrally managed for actions like tracking of consumed and produced energy, computation of energy prices, immutable, and secure recording of all the information related to the energy transactions [40], [41]. In a Peer-to-peer (P2P) context, this management is decentralized and regulated among the "peers" participating to the energy network, which becomes a virtual energy power grid [42]-[44].

In a centralized architecture, communication between prosumers should be authorized by centralized servers and the set requirements for them increase with the number of prosumers [45]. Centralized architectures are, therefore, not easily scalable to account for an exponential increase of prosumers, which in turn produce high volumes of data at high frequency (i.e., every $60 \mathrm{~s}$ ). As a result, the integration costs to account for the magnitude of that increase would make a TMI not economically feasible [44]-[53].

The evaluation of the performances of a TMI is a complex task and different features can be considered, the most important being:

1) data security, because financial data is being exchanged;

2) data privacy, because energy data exchange can profile the user;

3) speed of financial transactions or transactions insertion rate, ideally a constant value or at least sublinear e.g., $O(\log N)$, with $N$ being the number of transactions;

4) resiliency to failures and data integrity;

5) small energy footprint: the system must not consume more energy.

Given a certain level of performance, $p$, related to the previously listed features, the costs which must be sustained to guarantee this level can represent a metric for the scalability, $S=\mathrm{f}(N, p)$, which can be reasonably be assumed as a monotone function of the number of prosumers $N$. By using $S$ as a metric for comparing the centralized and $\mathrm{P} 2 \mathrm{P}$ approaches, the centralization of all the operations required to assure the level of performance $p$ can become infeasible for a huge number of prosumers. P2P transactions are, instead, an order of magnitude cheaper than those in traditional systems based on a centralized 339 information center [43].

To recall a parallel to computer networks where the exchanged 341 value is a file (or a database), P2P reduces the costs of the 342 scaled system and avoids to install more centralized hardware. 343 The centralized servers represent single points of failure and 344 may represent easy targets for attacks from hackers. The server 345 loads and delay can be reduced by leveraging the capabilities 346 of P2P networks which inherently scale "well" with the num- 347 ber of connected devices. In computer networks and data center 348 management, a similar problem of performance arises whenever 349 an increased service demand takes place. Usually, the owner of 350 the data center has two options: vertical scaling versus hori- 351 zontal scaling. In the vertical scaling, the owner installs more 352 bare metal, i.e., by buying more hardware with increased per- 353 formance and maintenance costs. In the horizontal scaling, the 354 owner replicates its service on different (physical or logical) lo- 355 cations, thus reducing the maintenance costs (like redundancy, 356 failover, etc.).

To summarize, the main advantages of a P2P decentralized 358 architecture over a centralized one are in terms of scalability, 359 resiliency, adaptability, fault tolerance, security, and trust. In- 360 vestment and maintenance costs are also reduced due to the 361 adoption of hierarchical storage capacity and a sublinear cost of 362 ownership which grows as $O(\log M)$, where $M$ is the number of 363 nodes [46].

\section{B. Blockchain Technology Applied to Transactive} 365 Energy Systems

Since envisioning totally disconnected micro-grids from the 367 main Distribution System Operator (DSO) is still premature, a 368 more realistic deployment of transactive energy systems will 369 be a hybrid solution, where the regulation and the access to 370 the information required for the implementation of P2P energy 371 exchanges based on the TE only is managed by the DSO. For 372 example, the DSO could manage the access to the smart power 373 grid and grant access to the information system, but it would 374 not manage the energy transactions centrally. It means that: 1) 375 energy data transactions must be confirmed by peers by using 376 a sort of consensus protocol embedded in a shared execution 377 routine usually know as smart contract; and 2) transactions must 378 be stored securely within peers participating to the program. A 379 comprehensive review on $\mathrm{P} 2 \mathrm{P}$ and community-based markets 380 can be found in [47].

This is a call for the very popular technology named Dis- 382 tributed Ledger Technology (DLT), representing an abstraction 383 of the so popular blockchain technology.

By abusing of the terminology, P2P may refer to both the way 385 according to which energy transactions take place and to the in- 386 formation architecture supporting them. Accordingly, from the 387 point of view of the information architecture, DLT is also a 388 $\mathrm{P} 2 \mathrm{P}$ based architecture and so it seems a natural candidate to 389 implement a TE system based on P2P energy exchanges (P2P- 390 $\mathrm{TE})$. In general, the term $\mathrm{P} 2 \mathrm{P}$ network is used when referring 391 to the information infrastructure (e.g., Internet) and P2P-TE 392 when discussing about the logical interaction among the peers 393 which trade and/or exchange energy resources. Concerning the 394 
type of access to the P2P network, the terms "permissionless" and "permissioned" are used. A permissionless architecture is a public network where everyone can participate without any special authentication/authorization mechanism. All peers are anonymous and untrusted. In a permissioned architecture, the access to the network must be granted (for example by registering one's identity to a central data center), although no central action interferes during the information exchange among peers (e.g., Skype could be thought as a permissioned P2P network). In a permissioned architecture, peers share some kind of trust and the identity is not completely anonymous. In the following subsection, a permissioned $\mathrm{P} 2 \mathrm{P}$ for the proposed P2P-TE system will be considered.

As stated before, to enable P2P energy transactions between prosumers and active users within a LEM context, DLT appears to be a promising solution [48], [49], as it avoids the need of an intermediator and can guarantee near real-time transactions. Currently, there are many type of DLT. The most popular has been that based on blocks-chain, where groups of transactions are stored in blocks of data chained one after another in order to make nearly impossible data forgery [50]. The storage of the transactions data into the blocks is secured by cryptographic functions and industry level security methodologies (e.g., signature based on the public-key paradigm) [51], [52]. The blockchain is stored globally within the network of participating peers and it is a virtuous application of decentralization algorithms. In the Internet universe, popular applications based on decentralized communication of information are Bittorrent for sharing files, and Skype, for sharing contacts. In the blockchain universe, the shared item is essentially some kind of "value." This value can be virtual coin or crypto currencies, smart contracts, virtual goods, agreements between untrusted parties. While in the centralized approach, the participating parties give trust one another to the central authority (e.g., in the case of a bank institution), in P2P untrusted parties use a mechanism to reach some kind of trust. This trust must at least guarantee:

1) anonymity;

2) impossibility to repudiate a transaction once it has been saved;

3) very low probability of forgery of saved data;

4) resiliency to possible attacks, like the byzantine attack where a transaction is authorized to be saved even if it should not.

It should be noted that points 2) and 3) are generally guaranteed by using cryptographic hashes which define each block and chaining of all blocks. In this way, transaction forgery would mean being able to modify all the chain. This is not a new concept, as it was popularized in 2009 when blockchain technology was used by the Bitcoin platform for cryptocurrency to enable secure virtual transactions [52].

That said, blockchain enables P2P transactions in a decentralized way, which perfectly fits with the idea of making energy transactions among the prosumers in a LEM context [53], [54], without the need of a central authority, such as the DSO in traditional distributed energy networks. Under these circumstances, a blockchain-based DLT might manage hundreds or even thousands of smart contracts [55] in near-real time and with no obstacle due to data center design and maintenance. However, 452 the original algorithm found in DLT like Bitcoin is able to cope 453 with a maximum of seven transactions per second [56]. For this 454 purpose, other alternatives have arisen, such as Ethereum [57], 455 which can handle tens of energy transactions per second, or 456 Hyperledger [58], [59], which can cope with hundreds of trans- 457 actions per second and has the additional advantage of being a 458 scalable solution, which makes it very suitable for smart con- 459 tracts [60]. A comparison between Bitcoin and Ethereum can be 460 found in [61], a comparison between Ethereum and Hyperledger 461 is given in [62], while a comparison between all current DLT- 462 based platforms used for TE in microgrids is carried out in [63]. 463

An example of an on-going project aimed at experimenting 464 blockchain-based P2P energy trading is transactive Grid [64], 465 [65], where prosumers in a small community in Brooklyn (USA) 466 can buy and sell energy from each other using Ethereum plat- 467 form for smart contracts. Another example is the UK company 468 Electron [66], which has used blockchain technology to cre- 469 ate an open-source platform for providing truthful metering. A 470 comparison between current projects on $\mathrm{P} 2 \mathrm{P}$ energy trading can 471 be found in [67].

\section{Consensus Protocols}

In a LEM with blockchain-based DLT, since there is no central 474 authority which manages the energy transactions, all prosumers 475 (or nodes from a system viewpoint) must agree upon a financial 476 energy transaction before storing it into the blockchain. The va- 477 lidity of a new transaction (or block, i.e., group of transactions) 478 holds if and only if a consensus is reached among all nodes [68]. 479 Consensus protocols are a set of algorithms and structured data 480 well known in many engineering fields, such as Computer Sci- 481 ence and Signal Processing. The key properties or requirements 482 of a consensus protocol are [69] as follows. 483

1) Safety: nodes that take part in a consensus produce the 484 same outputs according the protocol rules. 485

2) Liveness: all healthy nodes take part in consensus will 486 produce a value.

3) Fault tolerance: if a node that takes part in the consensus 488 fails, the consensus protocol can continue working. $\quad 489$

The most common consensus protocols in blockchain-based 490 DLTs are: Proof of work (PoW), Proof of stake (PoS) and Byzan- 491 tine Fault Tolerant (BFT). The PoW protocol [52] is used by 492 permissionless platforms (such as the aforementioned Bitcoin 493 or Ethereum platforms) in which a large number of untrusted 494 nodes seek for consensus to approve an energy transaction. PoW 495 algorithm appears to be the best option as far as private data 496 safety is concerned, because all nodes must solve a hard cryp- 497 tographic puzzle before adding a block into the chain, thus, 498 making the system impermeable to malicious trading [70]. The 499 validity of the "work" done is represented by the difficulty (in 500 terms of complexity and memory/CPU requirements) of the 501 cryptographic puzzle. The process to find a solution to this puz- 502 zle is called mining [71]. To gain the right to approve the new 503 block (and therefore to gain also an economic profit), one has to 504 invest in hardware: The more powerful hardware, the higher is 505 the probability to quickly solve the cryptographic puzzle. Once 506 
507 a solution is reached, the other peers can confirm the solution 508 (confirmation of the solution is much simpler than finding it).

509 Although, it has been emphasized that blockchain-based 510 DLT could enable TE exchanging among multiple DER 511 parties, care should be taken to the energy footprint of (any) 512 DLT technology. Indeed, a recent study reveals that Bitcoin's 513 blockchain energy footprint is similar to Ireland's average 514 electrical energy consumption [72], because of the energy 515 hunger of PoW algorithms. Moreover, the Bitcoin requires 516 over 3 GBs of compressed data to hold the entire blockchain, 517 obviously outstripping the capabilities of smart inverters or 518 transactive controllers [73]. Other aspects of DLT concern 519 the type of access to the IB (permissioned or permissionless 520 access, or a combination of both), real-time requirements for 521 the energy transaction, Immutability, Anonymity, and Security 522 (IAS) requirements of transactions [74].

523 In order to reduce the energy footprint in the Bitcoin-based 524 PoW protocol, the PoS protocol [75] substitutes the mining pro525 cess by the election of a node that acts as the evaluator. In other 526 words, the right to validate and insert a new block is granted 527 to that peer which can prove the ownership of some amount 528 of a variable called stake (in the cryptocurrency, the stake can 529 be also the currency itself). The selection based on stakes only 530 suffers from some problems. For example, in a pure PoS the 531 peer with the highest amount of stakes will gain a permanent 532 advantage. To overcome these and other shortcomings of PoS, 533 other variants have been proposed in the scientific literature. 534 For example, in the cryptocurrency world PeerCoin [76] and 535 Nxt [77] use a selection algorithm based on the concept of coin 536 age and a transformation of the stake size, respectively. How537 ever, as it will be detailed in the next section, PoS is a good 538 candidate to be used in the energy context. This manuscript will 539 sketch (see Section V) an architecture based on PoS by showing 540 that the basic problems of PoS can easily be avoided by using a 541 permissioned architecture and a hard-to-forge stake values.

542 A very interesting modified version of PoS is used in Solar543 Coin [78], a promising platform for selling solar energy through 544 certified production plants. In SolarCoin, every PV plant's owner 545 registers their PV installations thus becoming a prosumer. Then, 546 after verification of the identity and the details of the compo547 nents of the installation, the owner grants access to the platform 548 and receives a digital wallet. In SolarCoin, the software installed 549 into the user-side smart inverter communicates the energy pro550 duction only, and, subsequently, a block of "solar" transactions 551 is inserted into the DLT. For every MWh produced, the platform 552 pays back some "solar coins" and the transactions are stored into 553 the digital wallet by means of the blockchain. There is no cen554 tralized ledger for transactions. The BFT protocol [79] is used 555 to detect mismatches between the information shared among all 556 the nodes, thus avoiding the malfunction of the whole system. 557 A comprehensive explanation of PoW, PoS, and BFT proto558 cols can be found in [68]. A variant of PoS and BFT protocols 559 is the Tendermint protocol [80], which is a private one. Apart 560 from the aforementioned blockchain consensus protocols, in 561 the literature there are more examples. A comparison among 562 all the consensus protocols based on blockchain is detailed in 563 [81]. Recently, with the vision for a highly scalable DLT for the
IoTs, other concepts have emerged. For example, in the Tan- 564 gle algorithm, validation and insertion of transactions are based 565 on acyclic graphs and not chain of blocks. Tangle is the core 566 algorithm of the popular IOTA cryptographic token [82].

\section{Proposed Transactive MANAGEMENT InFRASTRUCtURE 568}

\section{A. Architecture}

The proposed system is a permissioned blockchain based 570 architecture where the consensus protocol is a modified version 571 of PoS which, in comparison, uses less than $0.001 \%$ of the power 572 of Bitcoin [78]. Therefore, it promotes energy efficiency and a 573 sustainable behavior.

The envisioned architecture, called Transactive Management 575 Infrastructure (TMI), represents the main novel contribution of 576 the paper and is depicted in Fig. 2.

One of the innovative contributions of the present work with 578 respect to the present literature is the attempt to establish the 579 baseline for a reference framework for blockchain-based TMI 580 that can be used by medium sized aggregators to manage LEMs. 581 Without any claim of superiority, from an extensive analysis of 582 the literature results that previous works do not provide tech- 583 nical details of their solutions. It is, therefore, not possible to 584 understand the implementation details of the blockchain-based 585 TMIs due to the lack of a detailed explanation of the software 586 implementation. Instead, in this paper details related to the im- 587 plementation of both the proposed TMI (based on MQTT +588 JSON) and of the proposed DLT technology are specified. The 589 adopted DLT technology makes use of a PoS scheme, within 590 a permissioned system where participants grant access to the 591 TMI. While the proposed scheme overcomes the energy foot- 592 prints many blockchains schemes suffer from, the consensus 593 protocol is based on a novel CPF that promotes the rational use 594 of energy resource also contributing to reduce power losses in 595 the distribution and transmission systems.

The TMI is a layered architecture which consists of three lay- 597 ers: 1) the aggregator owned data center, where the virtual ex- 598 change of energy is accomplished; 2) the communication layer, 599 consisting of all the components needed to let the TCRs com- 600 municate one other (this will contain also the Internet Cloud part 601 which rules the access of the TCRs to the DR-TE program); and 602 3 ) the user layer, where the TCRs execute the DR algorithms 603 and perform all the communication to the IT infrastructure. The 604 first layer regards a digital communication infrastructure. Basi- 605 cally, it can be thought as a virtualized set of servers which are 606 centrally managed by some central actor. The central actor can 607 be an aggregator, or some other authority like the DSO.

The only purpose of the central actor is to provide participants 609 of the LEM with the basic software and telecommunication net- 610 working components for the exchange of energy related data 611 (energy measurements, smart contract info and/or market trad- 612 ing information). It does not implement any other functions, like 613 energy transactions storage, validation of virtual "coins," etc., 614 because all these information are exchanged by means of the 615 DLT, i.e., by means of blockchain based smart contracts. $\quad 616$

Therefore, the role of the central actor is to manage the Trans- 617 active Management Platform (TMP), only. The core components 618 
TMI - Transactive Management Inftrastructure

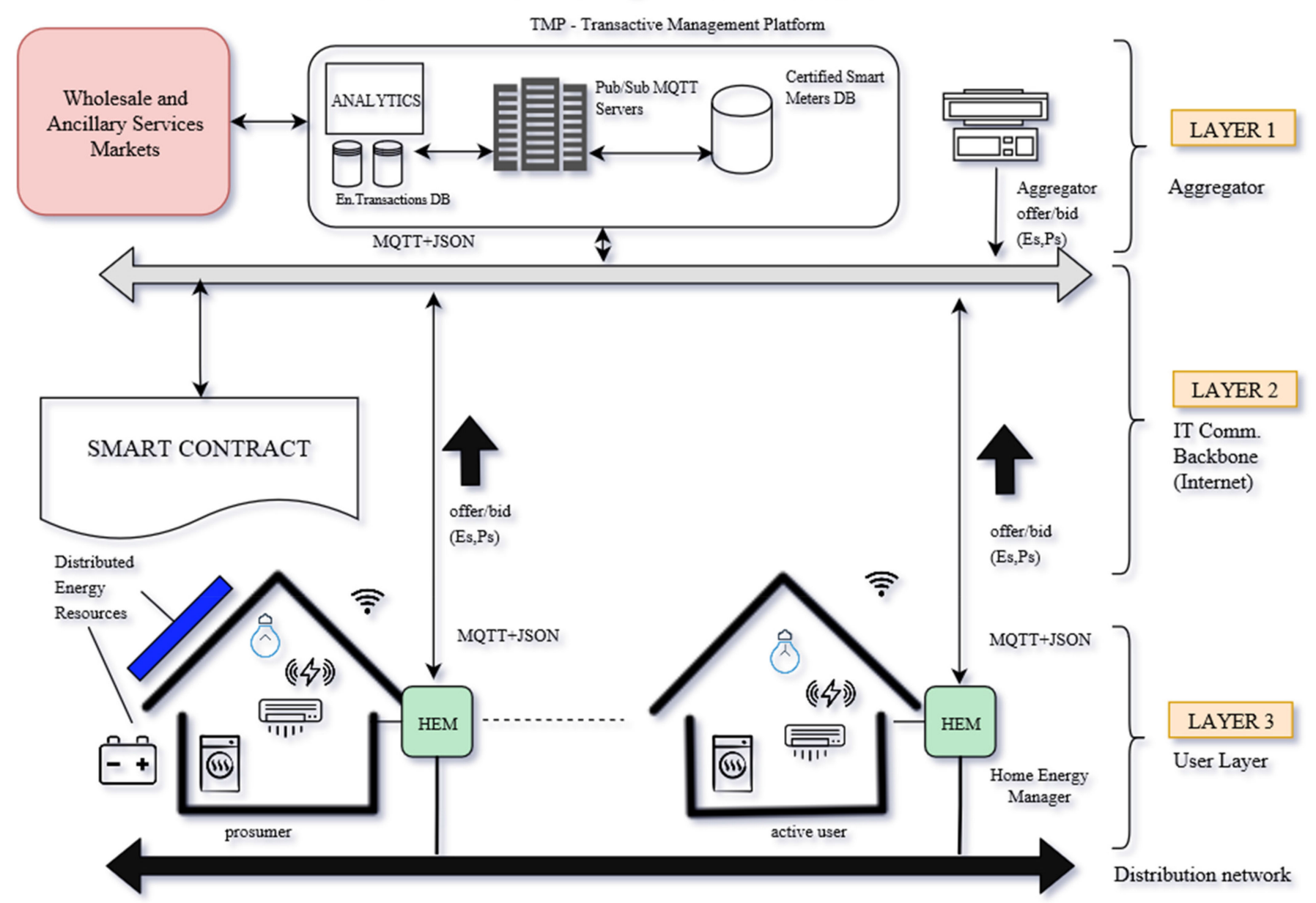

Fig. 2. Transactive Management Infrastructure (TMI): Proposed architecture to enable P2P transactive energy exchanges.

619 of the TMP are: 1) the Pub/Sub servers, 2) the Certified Smart 620 Meters DB (CSM-DB), and 3) the Analytics Component (AnC). 621 The Pub/Sub servers are needed for asynchronous and ubiq622 uitous communication among parties. They use a well-known 623 computer network design pattern based on the concept of 624 Publisher-Subscriber. Without entering into the details, it can 625 be explained by saying that participants subscribe to virtual in626 formation channels, usually called rooms or topic rooms, and 627 publish data on the subscribed channels. The role of the Pub/Sub 628 servers or brokers is to receive the publishers' data and broadcast 629 them to other participants on the same channel. In this context, 630 the participants are the prosumers and active users that gain ac631 cess to the platform through the HEM in a ubiquitous fashion, 632 i.e., without any sort of operation on the user appliances (e.g., 633 routers). The energy data is exchanged through the brokers by 634 using dedicated channels the HEM are subscribed to.

635 The network protocol used to handle the data publishing is 636 the Message Queue Telemetry Transport (MQTT), which is a 637 lightweight application protocol very popular among the IoT 638 community. It was conceived for embedded devices with con639 strained energy and computational resources. It also supports 640 Quality of Service (QoS) concepts. For example, in MQTT three QoS levels are conceived. QoS 0 refers to the case of 641 transmission without retransmission in case of packet loss. QoS 642 1 and 2 refer to guaranteed delivery of packets (e.g., this level 643 can be used to send critical commands to devices). The data for- 644 mat transported by the MQTT is the JavaScript Object Notation 645 (JSON), which is a ubiquitous data exchange format easily to 646 extend and to implement. It is based on a key-value structure. 647 For example, the following JSON snippet could represent the 648 measured temperature of a sensor:

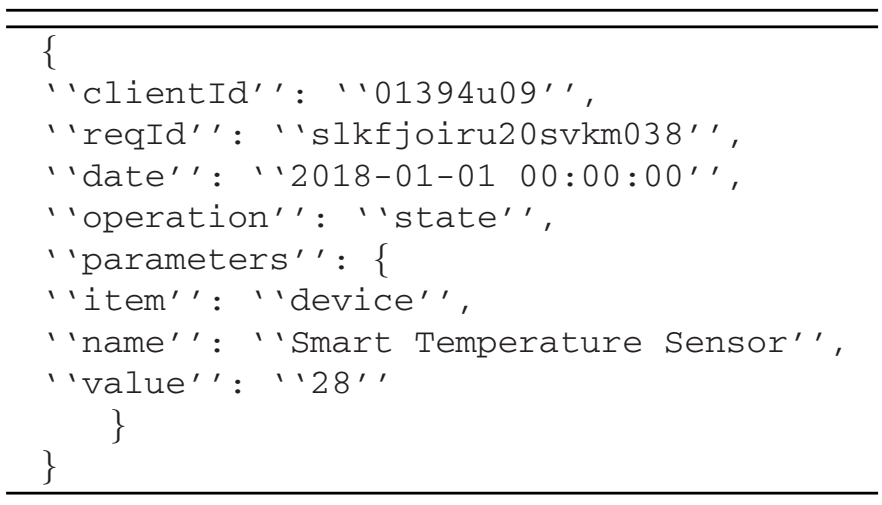




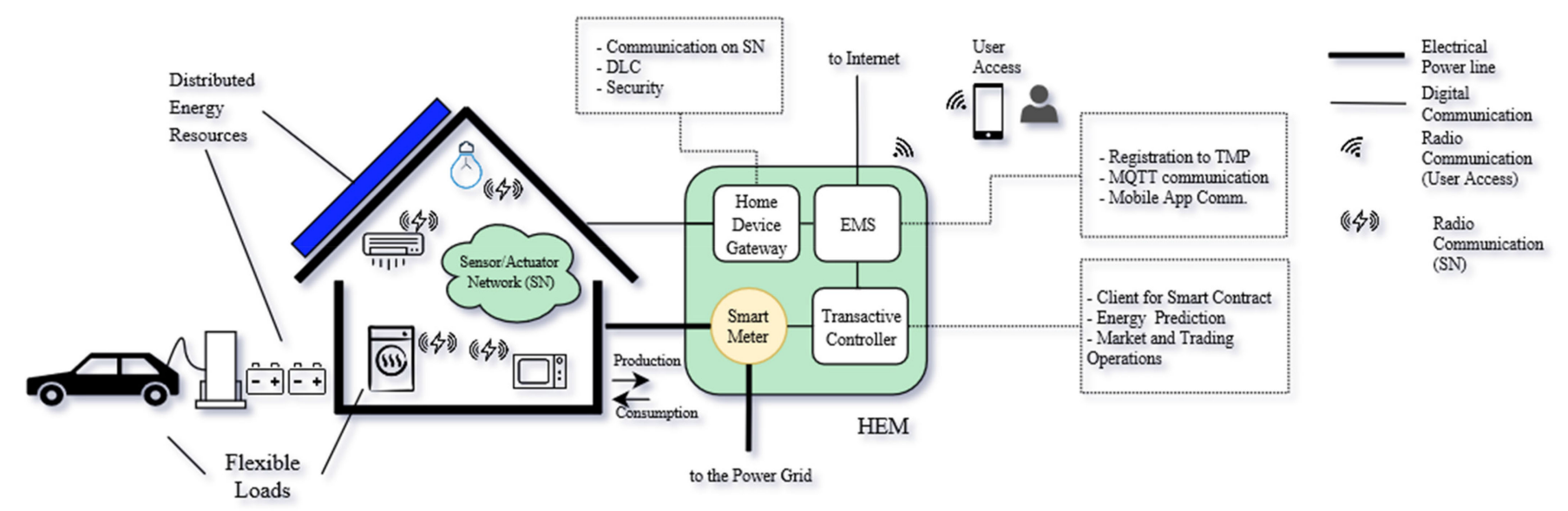

Fig. 3. Structure of the proposed home energy manager (HEM).

The CSM-DB contains users' information about verified

\section{B. Implemented Home Energy Manager}

Every participant to the LEM uses an HEM, which is a hard669 ware component (a microcomputer such as a Raspberry Platform) installed inside buildings or houses. As assumption, every participant, both prosumer and active user, has a smart meter, i.e., an energy meter which can communicate outside and in near-real time the energy data flow, for instance the energy production and energy costs of the building. This information is vital for the operation of the overall architecture and must be shared with the components of the HEM.

The purpose of the HEM is to let the user (prosumer or active user) access the TMP in order to act as a transactive agent within the LEM. In other words, the HEM is the interface between the prosumer/active user and the smart grid. The HEM is composed of three main parts, as shown in Fig. 3: a Home Device Gateway (HDG), a TCR and an Energy Management System (EMS).

The HDG is an interface for communication to the smart devices deployed within the house or the building. For example, a set of smart switches and temperature sensors which communicate by means of a wireless mesh networking protocol like Z-Wave or Zigbee or a combination of other ad-hoc sensor net88 work protocols [83]. The HDG provides the HEM with access to user's smart devices. After the access is granted, sensors and 689 actuators (switches, valves, dimmers, etc.) can transmit mea- 690 surements and receive control commands from the EMS. The 691 HDG is responsible for the very important aspect related to 692 information security and safety.

The TCR is the software engine needed to make decisions 694 about the energy trading operations. For instance, the TCR 695 makes energy bids and offers in order to buy or sell energy, 696 respectively, and receives information related to their accep- 697 tance/rejection in the LEM inside the VPP aggregator, as it will 698 be described more in details in the following sub-section. The 699 TCR is interfaced to the EMS, the HDG and the smart meter. 700 The EMS coordinates all the communication between the TCR 701 and the HDG toward the TMP. The main functions of the EMS 702 concern the registration of the HEM to the TMP and the com- 703 munication of energy and trading data to the shared channels 704 used by all participants.

\section{Smart Contract Deployment for Managing the Interactions 706 Between the VPP Aggregator and Its Aggregated Prosumers 707}

Even though the proposed transactive management infrastruc- 708 ture is general and can be used in different contexts and with 709 different players, in order to demonstrate a possible application, 710 a realistic scenario is presented.

It is assumed that a Virtual Power Plant (VPP) aggregator can 712 deliver services to the Transmission System Operator (TSO) by 713 participating to the ancillary services market.

The proposed architecture can be used to provide reliable 715 and speedy two-way communication, allowing the aggregator 716 to interface with its internal prosumers and with external parties 717 such as the DSO, the TSO or the market operator. 718

During the day-ahead or hour-ahead the aggregator provides 719 a generation/load schedule for the aggregated prosumers also 720 considering transmission network technical constraints under 721

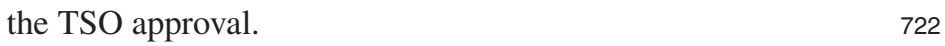

Day-ahead schedule is carried out in order to allow the aggre- 723 gator to effectively participate to the ancillary services market. 724 The schedule is based on historical data and on the forecasting 725 of the baseline electrical load for each prosumer also consider- 726 ing RES and electrical load forecasts. Moreover, the schedule 727 
should depend on which strategy the aggregator adopts for participating in the ancillary services market. This choice depends on the price and load forecasts within the electricity market and on the optimal time slots for selling/buying energy in the ancillary services market.

Prosumers associated to the VPP are supposed to be connected to the same electrical distribution network or feeder, so that the physical energy exchange determined by the aggregator schedule may take place while complying with distribution network technical constraints. The technical feasibility of the energy exchange is previously approved by the DSO, which, if technical problems are expected in the distribution networks, can ask the aggregator to modify its schedule.

If the aggregator cannot follow the scheduled day-ahead program in real-time, due to an energy deviation caused by errors in the forecast of electrical energy generated by RES or absorbed by loads, it makes an offer or a bid to all its aggregated prosumers in order to sell or to buy, respectively, the required electrical energy quantity at a determined price. The prosumers can react to the offer/bid of the aggregator by making their own bids/offers, respectively.

Indeed, when an offer/bid is made by the aggregator, a smart contract is deployed to the blockchain and an auction is started allowing prosumers to make offers/bids by means of their TCRs.

The smart contract program, which is a set of rules encoded into the blockchain, enables the execution of an auction to determine the accepted offers/bids that will give rise to trusted energy transactions in the LEM of the aggregator. The selection of the auction type encoded inside the smart contract is agreed between the aggregator and the prosumers [27].

For example, when the aggregator makes a bid, the offers of the prosumers having prices lower than the aggregator's bid price are selected in increasing order of price until the quantity of energy required from the bid is reached. After that the transactions are completed and verified by the smart meters of the prosumers the cryptocurrencies exchanges are authorized.

\section{Transactive Controller Operation to Manage the}

\section{Battery of a Residential Prosumer}

Even though different controllable electrical loads can be managed by the TCR as flexibility resources, such as HVAC, hot water heater, dish washer, washing machine, dryer, etc. [33], the use of the battery as flexibility energy source instead of controllable electrical loads makes the provision of the energy flexibility service more acceptable by the prosumers since it does not interfere with the normal activities and habits of the prosumer. Batteries group ensures a higher degree of reliability, indeed, because bidirectional power converters can charge the battery both from the main electrical grid and from the local PV source as well. On these bases, and in order to detail the bidding/offering process, it has been supposed that the battery is the only flexibility resource for a prosumer. As previously stated, the TCR can alternatively make an offer (to discharge the battery) or a bid (to charge the battery).

Different parameters, including the battery energy capacity, the degradation cost of the battery, the charging/discharging

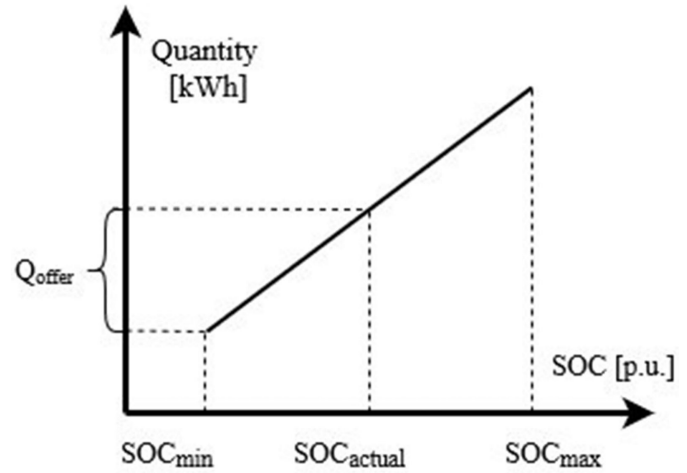

Fig. 4. Quantity offering curve.

rate limits and the State of Charge (SOC) are considered by 783 the proposed TCR to determine the bid/offer quantity and price. 784 The quantity is determined considering the admissible range of 785 the SOC, the charging and discharging rates and the capacity of 786 the battery according to equations from (1) to (6).

Inequality (1) limits the SOC in its admissible range

$$
\mathrm{SOC}_{\text {min }} \leq \mathrm{SOC}_{t} \leq \mathrm{SOC}_{\max } \quad \forall t .
$$

The charging and discharging rates of the battery at time $t, 789$ $r_{t}^{\text {charge }}$ and $r_{t}^{\text {discharge }}$ should respect their maximum rate limits 790 as presented in the following:

$$
\begin{aligned}
r_{t}^{\text {charge }} & =\left(\mathrm{SOC}_{t}-\mathrm{SOC}_{t-1}\right) / \eta^{\text {charge }} \quad \forall t \\
r_{t}^{\text {discharge }} & =\left(\mathrm{SOC}_{t-1}-\mathrm{SOC}_{t}\right) \eta^{\text {discharge }} \quad \forall t \\
0 & \leq r_{t}^{\text {charge }} \leq r^{\text {charge,max }} \quad \forall t \\
0 & \leq r_{t}^{\text {discharge }} \leq r^{\text {discharge,max }} \quad \forall t
\end{aligned}
$$

where $\eta^{\text {charge }}$ and $\eta^{\text {discharge }}$ denote the charging and discharging 792 efficiencies of the battery, respectively.

The following equation describes the model considered for 794 assessing the $\mathrm{SOC}$ variations:

$$
\mathrm{SOC}_{t}=\mathrm{SOC}_{t-1}+\gamma_{t}^{B} \eta^{\text {charge }} \frac{E_{t}^{\text {ch }}}{\operatorname{Cap}^{B}}-\chi_{t}^{B} \frac{E_{t}^{\text {disch }}}{\eta^{\text {discharge }} \operatorname{Cap}^{B}} \forall t
$$

where $\mathrm{Cap}^{B}$ is the battery capacity.

The quantity offering and bidding curves are shown in Figs. 4797 and 5, respectively. It is worth noting that the quantity is pro- 798 portional or inversely proportional to the SOC, in the case of an 799 offer or a bid, respectively.

Although the price offering and bidding curves exhibit a be- 801 havior similar to the quantity curves, some additional limitations 802 should be considered. They have to take into account the degra- 803 dation cost of the battery when the TCR makes an offer and 804 in order to make the bid acceptable by the aggregator. In other 805 words, the bid price should be higher than the offer price decided 806 by the aggregator and the offer price should be always higher 807 than the degradation cost of the battery, calculated as described 808 in the following equations.

The degradation cost of the battery due to the operation in 810 discharge mode is calculated by

$$
\mathrm{Cost}_{t}^{\text {Degr }}=E_{t}^{\text {disch }} C d
$$




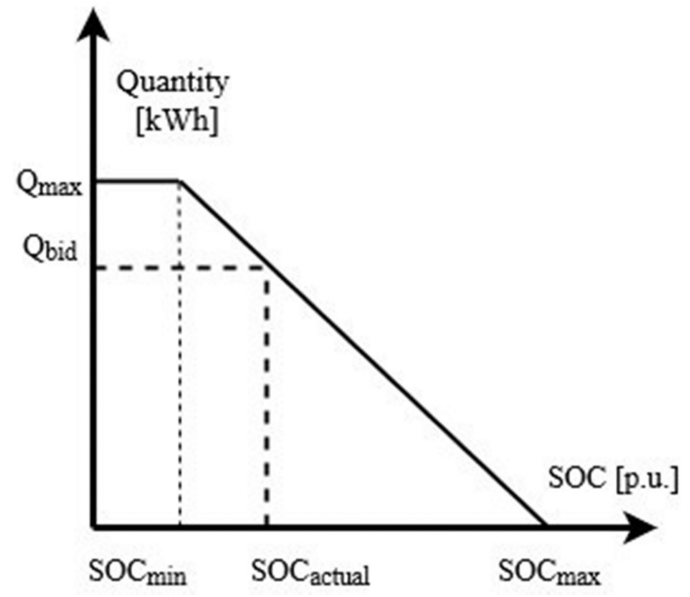

Fig. 5. Quantity bidding curve.

812 where $E_{t}^{\text {disch }}$ is the discharged energy of the battery. $C_{d}$ is the 813 cost of the battery in euros $/ \mathrm{kWh}$ dependent on the discharging 814 and is evaluated by the following:

$$
C_{d}=C_{\text {battery }} / L_{E T}
$$

815 where $C_{\text {battery }}$ is the capital cost of the battery and $L_{E T}$ is the 816 battery life time for the specific cycling regime.

\section{E. How the TMP works}

In the presented TMP architecture, the software which runs 819 inside the HEM, and specifically the TCR and the EMS, is 820 capable of doing following functions.

1) Making and receiving bids and offers when these are advertised on the common MQTT channel.

2) Communicating anonymously the ConsumptionProduction Function (CPF) value in order to preserve the user's privacy.

3) The consensus protocol is based on the value of stakes, where stakes are distributed according to the CPF values.

4) Implementing the blockchain to store energy transactions.

5) Digital wallets can be queried anytime.

The requirement about anonymity takes into account security needs. A user could be profiled by inspecting his/her energy transactions on public channels of the TMP, and this information could be used to track habits or daily behaviors. To protect the identity of users on public channels, e.g., the offer channel, every user is given a random address.

The software installed on the user-side smart inverter communicates the energy production, while a smart meter communicates the energy consumption. Using a function that combines the consumed and produced energy from RES is an omni-comprehensive incentive to a green economy as explained in Section V-F.

In the proposed TMP, prosumers can also decide to take part in an incentive program for improving the self-consumption of locally produced energy.

Different options are thus allowed for the prosumers, i.e., 1) Transactive Energy (TE); 2) Rewards (RE); or 3) RE + TE.
In the TE option the prosumers and active users can start an 847 auction process by making an offer or a bid in the LEM [84]. $\quad 848$

In the TE option the participants take part in energy ex- 849 changes. The key-value-based JSON structure for energy trans- 850 actions can be as follows:

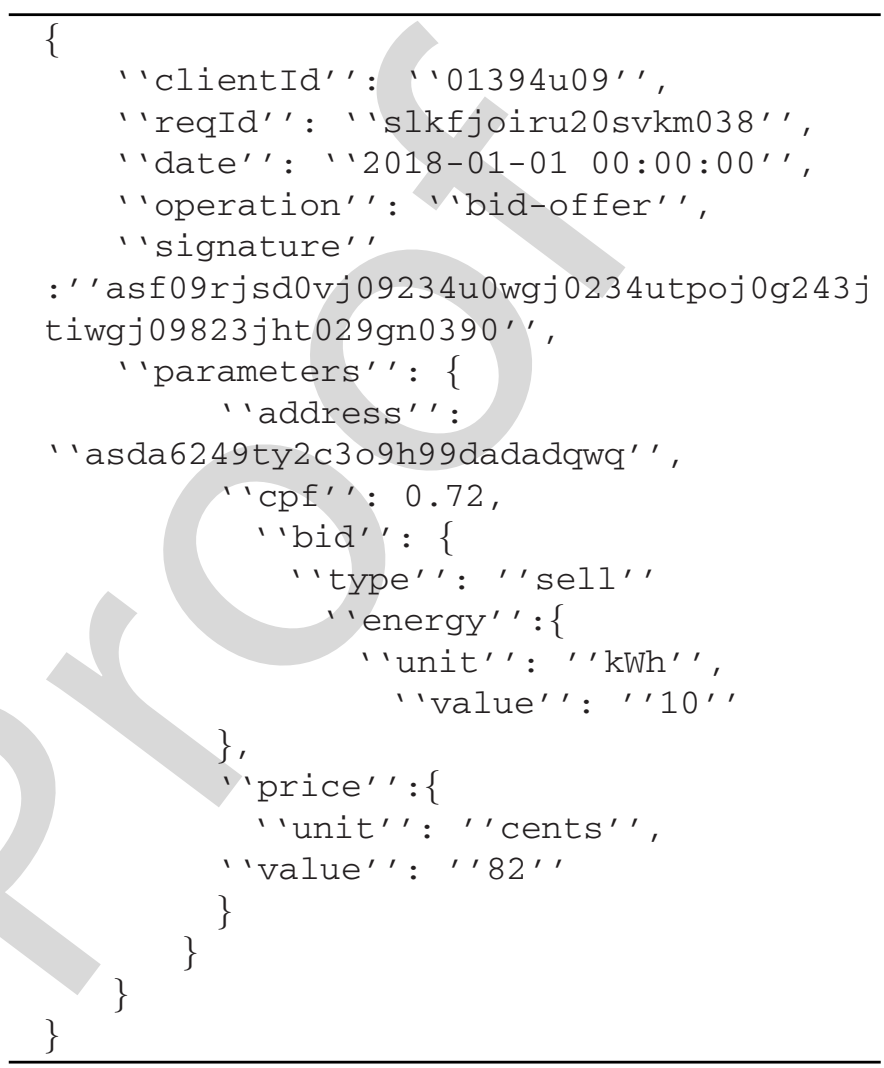

The signature is a cryptographic value generated by the EMS 852 and based on standard asymmetric encryption (public-private 853 keys). The public key of the EMS uniquely identifies the user and 854 it is stored in the CSM-DB. The EMS will sign every message 855 with the private key. The validity of the message can be checked 856 by verifying the signature embedded in the message. In the RE 857 mode, the TMP can verify the authenticity of the message. Other 858 forms of antiforgery of the value of the produced energy can be 859 applied by exploiting additional cryptographic tools. But the 860 system is supposed to be strictly coupled with the hardware of 861 the inverter, which is assumed to be hard to hack.

\section{F. Proof of Energy (PoE) Proposed Function}

In the TE mode, the bids and offers are managed by the smart 864 contracts registered into the blockchains and handled by a TCR, 865 as in [81]. Once the smart contract is validated, for example 866 whenever the bid or offer is going to be accepted by some 867 participant, the generator of the next block in the blockchain 868 must be chosen. The election of the next block generator is 869 based on a simplified PoS, which is named Proof of Energy 870 (PoE) and it is based on the following Consumption-Production 871 


$$
\mathrm{CPF}=\frac{1}{e^{|P-C|}} ; \quad 0<\mathrm{CPF} \leq 1
$$

873 where, for each prosumer, $P$ is the energy production from local 874 RES generators connected to the prosumer, such as a PV system, 875 and $\mathrm{C}$ is its energy consumption, i.e., the energy absorbed by 876 all the electrical loads including the energy storage. It should 877 be noted that if either $P>>C$ or $C>>P$, then the CPF tends 878 to 0 ; otherwise, if $P$ and $C$ are similar, then CPF tends to 1 . 879 The validator node will be the one which has the CPF closer 915 technologies such as Distributed Ledger Technology, Peer-to916 Peer transactive energy exchanges and Local Energy Markets 917 for achieving energy efficiency in modern transmission and dis918 tribution systems.

919 Considering that the traditional centralized energy systems 920 are no longer viable, peer-to-peer energy transactions based on 921 DLT and transactive controllers in LEMs represent the most 922 likely evolution for future smart grids, as confirmed by recent 923 pilot projects. A crucial point for the use of DLT is the selection 924 of a proper consensus protocol: as PoW consensus protocol is very energy demanding, new approaches such as PoS are 925 needed.

On these basis, a permissioned blockchain based architec- 927 ture, using an adapted version of $\mathrm{PoS}$ as consensus protocol is 928 proposed to achieve energy efficiency and sustainability. The 929 concept of proof of energy has been proposed as a modifi- 930 cation of the proof of stake protocol in order to increase the 931 self-consumption ratio of prosumers, thus contributing to power 932 losses reduction.

A new designed and implemented TMI is proposed and de- 934 scribed that can represent a baseline for a reference framework 935 for blockchain-based TMI based on smart contracts that can 936 be used to manage LEMs. The proposed infrastructure con- 937 sists of three layers, namely: aggregator layer, communication 938 layer, and user layer. The aim of the first layer is to manage 939 the TMP, which is based on the Pub/Sub servers, the Certified 940 Smart Meters and the Analytics Component. The second layer 941 uses Internet Cloud to communicate among the different agents. 942 The third layer consists of an HEM, which lets active users and 943 prosumers to access the TMP.

It should be pointed out that different challenges should be 945 addressed by future research activities in order to make P2P 946 transactive energy exchanges and LEMs a reality. First of all, 947 the preservation of privacy in blockchain-based architectures 948 represents a research challenge and solutions to ensure the pro- 949 sumer privacy by design should be researched. Even if P2P 950 based solutions can exhibit better scalability than centralized 951 ones, studies and real tests should be carried out to evaluate the 952 scalability of blockchain based architectures when the number 953 of prosumers significantly increases. Even though some solu- 954 tions have been recently proposed to improve the scalability of 955 blockchain based architectures, further researches are required 956 to identify new methods for improving scalability. Also, stan- 957 dardization and interoperability issues need to be investigated 958 when designing blockchain based architectures. 959

Concerning LEMs, future research activities should be car- 960 ried out to evaluate the impact of different markets and auc- 961 tion mechanisms on the power losses and technical constraints 962 of distribution and transmission systems. In addition, different 963 options for managing the interactions and mutual effects be- 964 tween LEMs and the wholesale market should be investigated, 965 while new rules are necessary to regulate the interactions be- 966 tween DSOs and the TSO. Further researcher activities are also 967 required to investigate the effects of transactive controllers on 968 consumers' behavior and their willingness to take part in LEMs. 969

\section{REFERENCES}

[1] Renewable Energy Policy Network for the 21st Century (REN21), Re- 971 newables 2017. Global status report, Tech. Rep., Mar. 2017. [Online]. 972 Available: http://www.ren21.net/. Accessed on: May 7, 2018.

[2] International Energy Agency (IEA), Market report series: renewables 974 2017. Analysis and forecasts to 2022, Tech. Rep., Oct. 2017. [Online]. 975 Available: https://www.iea.org/. Accessed on: May 7, 2018.

[3] International Renewable Energy Agency (IRENA), Global energy trans- 977 formation. A roadmap to 2050, Tech. Rep., Apr. 2018. [Online]. Available: 978 http://www.irena.org/. Accessed on: May 7, 2018.

[4] A. Y. Saber and G. K. Venayagamoorthy, "Plug in vehicles and renew- 980 able energy sources for cost and emission reductions," IEEE Trans. Ind. 981 Electron., vol. 58, no. 4, pp. 1229-1238, Apr. 2011. 
[5] P. Siano, "Demand response and smart grids - A survey," Renew. Sust. Ener. Rev., vol. 30, pp. 461-478, Feb. 2014.

[6] R. A. Verzijlbergh, L. J. de Vries, G. P. J. Dijkema, and P. M. Herder, "Institutional challenges caused by the integration or renewable energy sources in the European electricity sector," Renew. Sust. Ener. Rev., vol. 75, pp. 660-667, Aug. 2017.

[7] J. Chen, F. N. Lee, A. M. Breipohl, and R. Adapa, "Scheduling direct load control to minimize system operation cost," IEEE Trans. Power Syst., vol. 10, no. 4, pp. 1994-2001, Nov. 1995.

[8] N. Lu, "An evaluation of the HVAC load potential for providing load balancing service," IEEE Trans. Smart Grids, vol. 3, no. 3, pp. 1263 1270, Sep. 2012

[9] D. Alahakoon and X. Yu, "Smart electricity meter data intelligence for future energy systems: a survey," IEEE Trans. Ind. Informat., vol. 12, no. 1, pp. 425-436, Feb. 2016.

10] H. Arasteh et al., "IoT-based smart cities: A survey," in Proc. 16th Int. Conf. Environ. Elect. Eng., Florence, Italy, Jun. 2016, vol. 1, pp. 1-6.

1] C.-H. Lo and N. Ansari, "The progressive smart grid system from both power and communications aspects," IEEE Commun. Surveys Tuts., vol. 14, no. 3, pp. 799-821, Sep.-Dec. 2012.

] H. Farhangi, "The path of the smart grid," IEEE Power Eng. Mag., vol. 8, no. 1, pp. 19-28, Jan./Feb. 2010.

S. Soft, Power System Economics - Designing Markets for Electricity. New York, NY, USA: Wiley, 2002, pp. 2-29.

4] A. Ramos, C. De Jonghe, V. Gómez, and R. Belmans, "Realizing the smart grid's potential: defining local markets for flexibility," Utilities Policy, vol. 40, pp. 26-35, Jun. 2016.

5] C. Block, D. Neumann, and C. Weinhardt, "A market mechanism for energy allocation in micro-chp grids," in Proc. IEEE 41st Annu. Hawaii Int. Conf. System Sci., Waikoloa, HI, USA, Jan. 2008, vol. 1, pp. 1-11.

6] E. Mengelkamp and C. Weinhardt, "Clustering household preferences in local energy markets," in Proc. ACM 9th Int. Conf. Future Energy Syst. (e-Energy), Karlsruhe, Germany, Jun. 2018, pp. 538-543.

C. Rosen and R. Madlener, "An auction design for local reserve energy markets,” Decis. Supp. Syst., vol. 56, pp. 168-179, Dec. 2013.

8] C. Eid et al., "Market integration of local energy systems: is local energy management compatible with European regulation for retail competition?," Energy, vol. 114, pp. 913-922, Nov. 2016.

9] F. Hvelplund, "Renewable energy and the need for local energy markets," Energy, vol. 31, no. 13, pp. 2293-2302, Oct. 2006.

] H. Lund and E. Münster, "Integrated energy systems and local energy markets," Energy Policy, vol. 34, no. 10, pp. 1152-1160, Jul. 2006

1] B. A. Bremdal, P. Olivella-Rosell, J. Rajasekharan, and I. Ilieva, "Creating a local energy market," in Proc. 24th Int. Conf. Exhib. Elec. Dist., Glasgow, U.K., Jun. 2017, vol. 1, pp. 2649-2652.

22] P. Sjöstrand and R. Zäther, "Design and modelling of a local energy market - A case study of Chalmers campus," M.S. thesis, Dept. Elect. Eng., Chalmers Univ. Technology, Gothenburg, Sweden, 2017.

23] F. Teotia and R. Bhakar, "Local energy markets: Concept, design and operation," in Proc. Nat. Power Syst. Conf. (NPSC), Bhubaneswar, India, Dec. 2016, vol. 1, pp. 1-6.

24] S. Grijalva and M. U. Tariq, "Prosumer-based smart grid architecture enables a flat, sustainable electricity industry," in Proc. IEEE PES Innov Smart Grid Tech., Anaheim, USA, Jan. 2011, vol. 1, pp. 1-6.

25] A. Abidin, A. Aly, S. Cleemput, and M. A. Mustafa, "Secure and privacyfriendly local energy trading and billing in smart grid," Comp. Res. Rep., vol. 1, pp. 1-13, Jan. 2018.

26] H. Vogt, H. Weiss, P. Spiess, and A. P. Karduck, "Market-based prosumer participation in the smart grid," in Proc. 4th IEEE Int. Conf. Digit. Ecosystems Technologies, Dubai, United Arab Emirates, Apr. 2010, vol. 1, pp. 592-597.

27] S. Chen and C.-C. Liu, "From demand response to transactive energy: State-of-the-art," J. Mod. Power Syst. Clean Energy, vol. 5, no. 1, pp. 1019, Jan. 2017

28] GridWise Architecture Council, GridWise Transactive Energy Framework, Tech. Rep., Jan. 2015. [Online] Available: https://www. gridwiseac.org/. Accessed on: May 14, 2018.

[29] F. Rahimi, "Transactive energy framework for bilateral energy imbalance management," in Proc. GridWise Architecture Council Meet., Dec. 2013., [Online] Available: https://www.gridwiseac.org/ Accessed on: May 14, 2018

0] D. P. Chassin, "Multi-scale transactive control in interconnected bulk power systems under high renewable energy supply and high demand response scenarios," Ph.D. dissertation, Dept. Mech. Eng., Univ. of Victoria, Victoria, Canada, 2017.
[31] J. Hu, G. Yang, K. Kok, Y. Xue, and H. W. Bindner, "Transactive control: A 1058 framework for operating power systems characterized by high penetration 1059 of distributed energy resources," J. Mod. Power Syst. Celan Energy, vol. 5, 1060 no. 3, pp. 451-464, May 2017

[32] W. Shi, N. Li, X. Xie, C.-C. Chu, and R. Gadh, "Optimal residential 1062 demand response in distribution networks," IEEE J. Sel. Areas Commun., 1063 vol. 32, no. 7, pp. 1441-1450, Jul. 2014.

[33] P. Siano and D. Sarno, "Assessing the benefits of residential demand 1065 response in a real time distribution energy market," App. Ener., vol. 161, 1066 pp. 533-551, Jan. 2016.

[34] A. K. Bejestani, A. Annaswamy, and T. Samad, "A hierarchical transactive 1068 control architecture for renewables integration in smart grids: analytical 1069 modelling and stability," IEEE Trans. Smart Grids, vol. 5, no. 4, pp. 2054- 1070 2065, Jul. 2014

1071

[35] V. C. Güngör et al., "Smart grid technologies: Communication technolo- 1072 gies and standards," IEEE Trans. Ind. Informat., vol. 7, no. 4, pp. 529-539, 1073 Nov. 2011.

[36] Z. Liu, Q. Wu, S. Huang, and H. Zhao, "Transactive energy: A review of 1075 state of the art and implementation," in Proc. IEEE Manchester PowerTech, 1076 Manchester, U.K., Jun. 2017, vol. 1, pp. 1-6.

[37] M. Sandoval and S. Grijalva, "Future grid business model innovation: 1078 Distributed energy resources services platform for renewable energy in- 1079 tegration," in Proc. Asia-Pacific Conf. Comput. Aided Syst. Eng., Quito, 1080 Ecuador, Jul. 2015, vol. 1, pp. 72-77.

[38] National Energy Technology Laboratory, Pacific Northwest Smart 1082 Grid Demonstration Project. Volume 1: Technology Performance, U.S. 1083 Department of Energy, Tech. Rep., Jun. 2015. [Online] Available: 1084 https://www.pnwsmartgrid.org/. Accessed on: May 14, 2018.

[39] S. Kesting and F. Bliek, "From consumer to prosumer: Netherland's pow- 1086 ermatching city shows the way," in Energy Efficiency: Towards the End 1087 of Demand Growth, 2013, pp. 355-373.

[40] J. J. Grainger and W. D. Stevenson, Power System Analysis. New York, 1089 NY, USA: McGraw-Hill, 1994, pp. 531-587.

[41] M. Shahidehpour, H. Yamin, and Z. Li, Market Operations in Electric 1091 Power Systems. Forecasting, Scheduling, and Risk Management. Hoboken, 1092 NJ, USA: Wiley, 2002, pp. 57-112.

1093

[42] T. Morstyn, N. Farrell, S. J. Darby, and M. D. McCulloch, "Using peer-to- 1094 peer energy-trading platforms to incentivize prosumers to form federated 1095 power plants," Nature Energy, vol. 3, no. 2, pp. 94-101, Feb. 2018.

[43] Q. H. Vu, M. Lupu, and B. C. Ooi, Peer-to-Peer Computing - Principles 1097 and Applications. Berlin, Germany: Springer, 2009, pp. 11-37.

1098

[44] J. Hu, R. Harmsen, W. Crijns-Graus, E. Worrell, and M. van den Broek, 1099 "Identifying barriers to large-scale integration of variable renewable elec- 1100 tricity into the electricity market: a literature review of market design," 1101 Renew. Sust. Energy Rev., vol. 81, no. 2, pp. 2181-2195, Jan. 2018.

[45] M. Andoni et al., "Blockchain technology in the energy sector: A system- 1103 atic review of challenges and opportunities," Renew. Sust. Energy Rev., 1104 vol. 100, pp. 143-174, Feb. 2019.

1105

[46] M. S. Ali, M. Vecchio, M. Pincheira, K. Dolui, F. Antonelli, and M. 1106 H. Rehmani, "Applications of blockchains in the Internet of Things: A 1107 comprehensive survey," IEEE Comm. Surv. Tutorials, in print.

[47] T. Sousa, T. Soares, P. Pinson, F. Moret, T. Baroche, and E. Sorin, "Peer- 1109 to-peer and community-based markets: A comprehensive review," Ren. 1110 Sust. Ener. Rev., vol. 1, Aug. 2018.

[48] A. Laszka, A. Dubey, M. Walker, and D. Schmidt, "Providing privacy, 1112 safety and security in IoT-based transactive energy systems using dis- 1113 tributed ledgers," in Proc. ACM 8th Intern. Conf. Internet Things, Linz, 1114 Austria, Oct. 2017, vol. 1, pp. 1-8.

[49] E. Mengelkamp, B. Notheisen, C. Beer, D. Dauer, and C. Weinhardt, "A 1116 blockchain-based smart grid: Towards sustainable local energy markets," 1117 Comput. Sci. Res. Dev., vol. 33, no. 1/2, pp. 207-214, Feb. 2018.

[50] P. Raj and G. C. Deka, "Blockchain technology: Platforms, tools and use 1119 cases," Adv. Comput., vol. 111, pp. 1-278, Sep. 2018.

[51] K. Townsend, "Blockchain technology impact on energy market trans- 1121 formation: Secured distributed energy transactions in the cloud," Geo. L. 1122 Tech. Rev., vol. 1, no. 2, pp. 469-476, Apr. 2017.

[52] S. Nakamoto, Bitcoin: A Peer-to-Peer Electronic Cash System, 1124 Mar. 2009. [Online] Available: https://bitcoin.org/. Accessed on: May 16, 1125 2018.

[53] R. Shandross, S. Chandler, and E. Gilbert, "The realities of peer-to-peer 1127 transactive energy using blockchain," in Proc. Transactive Ener. Syst. 1128 Conf., Portland, USA, Jun. 2017, pp. 1-12.

[54] E. Münsing, J. Mather, and S. Moura, "Blockchains for decentralized 1130 optimization of energy resources in microgrid networks," in Proc. IEEE 1131 Conf. Control Tech. Appl., Kohala Coast, USA, Aug. 2017, pp. 2164-2171. 1132 
1133 [55] K. Christidis and M. Devetsikiotis, "Blockchains and smart contracts for 1134 1135 1136 1137 1138 1139 1140 1141 1142 1143 the Internet of Things," IEEE Access, vol. 4, pp. 2292-2303, May 2016.

6] K. Croman et al., "On scaling decentralized blockchains," in Proc. Int. Conf. Financial Crypt. Data Sec (FC), Lecture Notes Comp. Sci., Berlin, Germany: Springer, Aug. 2016, vol. 9604, pp. 106-125.

57] G. Wood, "Ethereum: A secure decentralised generalised transaction ledger," Ethereum Project Yellow Paper, Apr. 2017, pp. 1-32.

58] Hyperledger, Hyperledger architecture. Volume 1: introduction to hyperledger business blockchain design philosophy and consensus, Tech. Rep., Aug. 2017. [Online] Available: https://www.hyperledger.org/. Accessed on: May 16, 2018.

59] Hyperledger, Hyperledger architecture. Volume 2: smart contracts, Tech. Rep., Apr. 2018. [Online] Available: https://www.hyperledger.org/. Accessed on: May 16, 2018.

60] M. Vukolic, "The quest for scalable blockchain fabric: Proof-of-work vs. BFT replication," in Proc. Int. Workshop Open Problems Netw. Security, Zurich, Switzerland, Oct. 2015, pp. 112-125.

61] D. Vujicic, D. Jagodic, and S. Randic, "Blockchain technology, Bitcoin, and Ethereum: A brief overview," in Proc. IEEE 17th Int. Symp. INFOTECH-JAHORINA, East Sarajevo, Bosnia and Herzegovina, Mar. 2018, pp. 1-6.

62] P. Sajana, M. Sindhu, and M. Sethumadhavan, "On blockchain applications: Hyperledger Fabric and Ethereum," Int. J. Pure App. Math., vol. 118, no. 18, pp. 2965-2970, Feb. 2018.

$63]$ M. L. Di Silvestre, P. Gallo, M. G. Ippolito, E. R. Sanseverino, and G. Zizzo, "A technical approach to the energy blockchain in microgrids," IEEE Trans. Ind. Informat., vol. 14, no. 11, pp. 4792-4803, Nov. 2018.

64] Det Norske Veritas (DNV) GL, The Brooklyn Microgrid. Blockchainenabled Community Power, Jan. 2018. [Online] Available: https://lo3energy.com/. Accessed on: May 26, 2018.

65] E. Mengelkamp, J. Gärttner, K. Rock, S. Kessler, L. Orsini, and C. Weinhardt, "Designing microgrid energy markets: a case study: The Brooklyn microgrid," App. Ener, vol. 210, pp. 870-880, Jan. 2018.

66] J. Hailstone, Electron Reveals Blockchain Energy Platform, May 2017. [Online] Available: http://www.electron.org.uk/. Accessed on: May 26, 2018.

67] C. Zhang, J. Wu, C. Long, and M. Cheng, "Review of existing peer-topeer energy trading projects," Ener. Procedia, vol. 105, pp. 2563-2568, May 2017.

[68] J. Debus, Consensus Methods in Blockchain Systems, FSBC Working Paper, May 2017. [Online] Available: http://www.fs-blockchain.de/. Accessed on: May 26, 2018.

69] A. Baliga, Understanding Blockchain Consensus Models, Tech. Rep., Apr. 2017. [Online] Available: https://www.semanticscholar.org/. Accessed on: May 26, 2018.

70] K. Kvaternik et al., "Privacy-preserving platform for transactive energy systems," in Proc. Middleware Conf., Las Vegas, NV, USA, Dec. 2017, pp. 1-6.
[71] J. Han, M. Kamber, and J. Pei, Data Mining: Concepts and Techniques. 1181 3rd ed. San Francisco, CA, USA: Elsevier, 2012, pp. 1-35. 1182

[72] K. J. O'Dywer and D. Malone, "Bitcoin mining and its energy footprint," 1183 in Proc. Irish Signals Syst. Conf., China-Ireland Int. Conf. Inf. Comm. 1184 Tech., Limerick, Ireland, Jun. 2014, pp. 1-6. 1185

[73] P. Eremy, "Bitcoin market data," Jan. 2018, [Online] Available: 1186 https://github.com/. Accessed on: May 29, 2018.

[74] AGL Energy Limited, Peer-to-peer distributed ledger technology assess- 1188 ment. Virtual peer-to-peer energy trading using distributed ledger tech- 1189 nology: comprehensive project assessment report, Tech. Rep., Oct. 2017., 1190 [Online] Available: https://arena.gov.au/. Accessed on: May 29, 2018.1191

[75] N. Courtois, On the longest chain rule and programmed self-destruction 1192 of crypto currencies, Tech. Rep., Dec. 2014. [Online] Available: 1193 https://arxiv.org/. Accessed on: May 29, 2018.

1194

[76] S. King and S. Nadal, PPCoin: Peer-to-Peer Crypto-Currency with 1195 Proof-of-Stake. Peercoin Whitepaper, Aug. 2012. [Online] Available: 1196 https://peercoin.net/. Accessed on: Jun. 25, 2018.

[77] Nxt community, Nxt Whitepaper, Jul. 2014. [Online] Available: 1198 https://bravenewcoin.com/. Accessed on: Jun. 25, 2018.

[78] SolarCoin, SolarCoin - A Blockchain-Based Solar Energy Incentive, 1200 Apr. 2018. [Online] Available: https://solarcoin.org/. Accessed on Jun. 1201 $25,2018$.

1202

[79] M. Castro and B. Liskov, "Practical Byzantine fault tolerance and proac- 1203 tive recovery," ACM Trans. Comp. Syst., vol. 20, no. 4, pp. 398-461, 1204 Nov. 2002.

[80] J. Kwon, "Tendermint: Consensus without mining," Oct. 2016. [Online] 1206 Available: https://tendermint.com/. Accessed on: Jun. 25, 2018.

[81] N. Chalaemwongwan and W. Kurutach, "State of the art and challenges 1208 facing consensus protocols on blockchain," in Proc. Int. Conf. Inform. 1209 Networking, Chiang Mai, Thailand, Jan. 2018, pp. 957-962. 1210

[82] S. Popov, The Tangle. IOTA Whitepaper, Oct. 2017. [Online] Available: 1211 http://iotatoken.com/. Accessed on: Jun. 25, 2018.

[83] Silicon Labs Whitepaper, The Wireless Protocols Tying Together the In- 1213 ternet of Things, 2018. [Online] Available: https://www.silabs.com/. Ac- 1214 cessed on: Jun. 25, 2018.

[84] A. Hahn, R. Singh, C-C Liu, and S. Chen, "Smart contract-based campus 1216 demonstration of decentralized transactive energy auctions," in Proc. IEEE 1217 Power \& Energy Soc. Inn. Smart Grid Tech. Conf., Washington, DC, USA, 1218 Apr. 2017, pp. 1-5.

Authors' photographs and biographies not available at the time of publication. 1220 